\title{
Importancia de la vena cística en la colecistectomía laparoscópica
}

\author{
Pablo Pinzón ${ }^{1,2,3}$, Luisa Trujillo G. ${ }^{1}$ y Camilo Ramírez G. ${ }^{1}$
}

\section{Importance of the cystic vein in laparoscopic cholecystectomy}

Aim: There are differences in the description of the cystic vein in the literature, often without considering it an important element. We consider it vital to describe the presence of the cystic vein as a significant element that can disturb the critical view of safety during cholecystectomies, and also cause mistakes. Materials and Method: Between January 1, 2014 and December 31, 2018, the presence of the cystic vein during the dissection of the cystohepatic triangle in cholecystectomies was evaluated. Results: 397 laparoscopic cholecystectomies were performed, in 30 cases additional elements were evidenced in the cystohepatic triangle, which required a careful dissection, which after following its path and when evaluating other anatomical repairs such as the Rouvière sulcus and the visualization of the biliary tract, it was considered due to its characteristics that it was a cystic vein in 8 cases (2\%). Discussion: The cystic vein is an anatomical structure whose existence must be kept in mind by the surgeon who performs laparoscopic cholecystectomy, because it can appear as a significant element altering the critical view of safety. Although in our series as a significant element it is $2 \%$, other series describe it with a much higher frequency. Conclusion: It is necessary to carry out more studies on the presence and importance as an anatomical repair due to its value to prevent bile duct injuries and as a factor of confusion in the critical safety view. Key words: gallbladder; cholecystectomy; veins.

\section{Resumen}

Objetivo: Existen diferencias en la descripción de la vena cística en la literatura, en muchas ocasiones sin considerarla un elemento importante. Consideramos que es de vital importancia describir la presencia de la vena cística como elemento significativo que puede alterar la vista crítica de seguridad (VCS) durante las colecistectomías y asimismo, originar errores. Materiales y Método: Entre el $1^{\circ}$ de enero de 2014 hasta el 31 de diciembre de 2018, se evaluó la presencia de la vena cística durante la disección del triángulo hepatocístico en las colecistectomías. Resultados: 397 colecistectomías laparoscópicas fueron realizadas, en 30 casos se evidenciaron elementos adicionales en el triángulo hepatocístico que requirieron una disección prolija, la cual después de seguir su trayecto y al evaluar otros reparos anatómicos como el surco de Rouvière y la visualización de la vía biliar, se consideró debido a sus características que se trataba de una vena cística en 8 casos (2\%). Discusión: La vena cística es una estructura anatómica cuya existencia se debe tener en mente por parte del cirujano que realiza la colecistectomía laparoscópica, porque puede aparecer como un elemento significativo alterando la vista crítica de seguridad. Aunque en nuestra serie como elemento significativo es del $2 \%$, otras series la describen con una frecuencia mucho mayor. Conclusión: Es necesario realizar más estudios sobre la presencia e importancia como reparo anatómico debido a su valor para prevenir lesiones de la vía biliar y como factor de confusión en la vista crítica de seguridad. Palabras clave: vesícula biliar; colecistectomía; venas.

\section{Introducción}

El drenaje venoso de la vesícula biliar ha sido considerado clásicamente, formado por múltiples ramas venosas que se integran en la superficie del cuerpo y el cuello, y se encuentran en el tejido areolar entre la vesícula y el hígado para, posteriormente, drenar dentro de los segmentos portales intrahepáticos ${ }^{1}$. Se ha descrito como un drenaje a través de numerosas pequeñas venas (entre 2-20)
'Universidad del Rosario. Bogotá, Colombia. ${ }^{2}$ Hospital Universitario Mayor. Bogotá, Colombia. ${ }^{3}$ Hospital Universitario Barrios Unidos. Bogotá, Colombia.

Recibido 2020-01-03 y aceptado 2020-03-03

Correspondencia a: Dr. Camilo Ramírez G ramirezgiraldocamilo@gmail. com

ORCID: 0000-0002-19292299 
que desembocan en el lecho vesicular en una red de capilares hepáticos, no formándose una única vena cística $^{1-4}$.

Sin embargo, otros autores han mencionado que se puede formar una vena cística única o doble, siendo esto poco frecuente, la cual drena directamente a la rama portal derecha o a la vena porta ${ }^{1,5-7}$. Además, se ha reconocido un drenaje de acuerdo a las porciones siendo el del cuello vesicular y del conducto cístico por venas císticas, pequeñas y múltiples que pueden desembocar en la vena porta $\mathrm{y}$, por otro lado, el fondo y cuerpo vesicular que drenan a través del lecho vesicular en los sinusoides hepáticos ${ }^{8,9}$ (Figura 1).

De igual forma, los libros de cirugía no dan mucha importancia al drenaje venoso de la vesicular biliar, por ejemplo, en el texto de cirugía de Sabiston se comenta que el drenaje es solo por medio de la vena porta y Schwartz en Principios de Cirugía comenta que el drenaje es variable y generalmente no es paralelo a las arterias ${ }^{10-12}$.

No obstante, la presencia de una vena cística ha sido respaldada y demostrada con casos de trombosis portal donde no solo causa dilatación del drenaje venoso esplácnico o formación de circulación colateral sino también dilatación de la vena cística. Un hallazgo poco reconocido, pero descrito en pacientes con hipertensión portal ${ }^{13}$.

Satoh, en un estudio para evaluar la diseminación del cáncer de vesícula, evaluó el drenaje venoso de este órgano usando 52 hígados. Encontró en la ma-

Figura 1. Vena cística que drena cuello vesicular y conducto cístico atravesando triangulo hepatocístico para drenar en ramas portales, y múltiples venas císticas que drenan cuerpo y fondo vesicular drenando en el lecho vesicular.

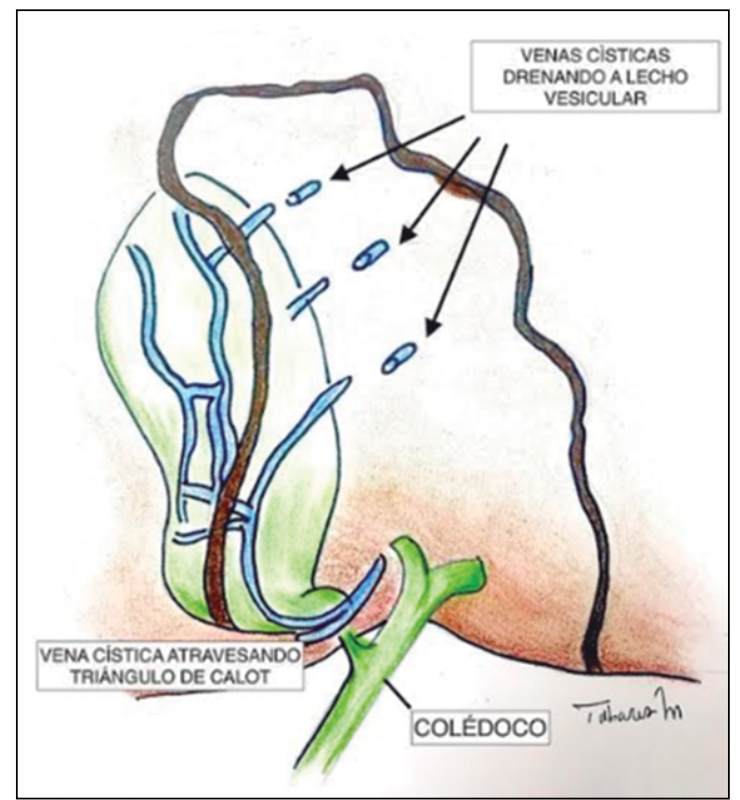

yoría de los casos que el drenaje venoso iba a la circulación portal hepática y 1 caso drenó directamente a la vena porta. Adicionalmente, encontró en 38 casos que las venas císticas atravesaban el triángulo hepatocístico con la arteria cística y terminaban en ramas portales, variando estas últimas ${ }^{14}$.

Samal y cols, realizaron un estudio donde evaluaron retrospectivamente 93 colecistectomías laparoscópicas encontrando la vena cística única o múltiple atravesando el triángulo hepatocístico en el $86 \%$ de los $\operatorname{casos}^{15}$.

Identificar la presencia de la vena cística en las colecistectomías laparoscópicas se hace importante, debido a que su presencia puede alterar la ventana crítica de seguridad que es una de las estrategias usadas para evitar lesiones de la vía biliar, por lo cual el objetivo de este trabajo es identificar la presencia de la misma.

\section{Materiales y Métodos}

Se realizó un análisis retrospectivo de las colecistectomías laparoscópicas realizadas entre el $1^{\circ}$ de enero de 2014 hasta el 31 de diciembre de 2018. Fueron incluidos todos los pacientes a los que se realizó colecistectomía de forma programada o de urgencia secundario a patología biliar benigna. Se excluyeron los pacientes que requirieron conversión a colecistectomía abierta, independiente de la causa. Se evaluó la presencia de la vena cística durante la disección del triángulo hepatocístico en estas colecistectomías.

\section{Resultados}

397 colecistectomías laparoscópicas fueron realizadas en un periodo de 5 años, por un cirujano, evaluando en cada una de ellas la presencia de vena cística.

La media de edad de los pacientes que se les realizó el procedimiento fue 58,5 años $273(68,7 \%)$ de los pacientes eran mujeres, dentro de los hallazgos intraoperatorios $302(76 \%)$ tenían cambios inflamatorios de la vesícula biliar, el resto presentaba únicamente colelitiasis. Dentro de las comorbilidades el $45(11,3 \%)$ pacientes eran diabéticos, 174 (43,8\%) eran hipertensos y únicamente $3(0,7 \%)$ pacientes eran cirróticos (Child A).

Se siguieron las estrategias para evitar lesión de vía biliar, principalmente, la VCS. Sin embargo, en 30 casos se evidenciaron elementos adicionales en el triángulo hepatocístico, que requirieron una disec- 
ción prolija, la cual después de seguir su trayecto y al evaluar otros reparos anatómicos como el surco de Rouvière y la visualización de la vía biliar, se consideró debido a sus características que se trataba de una vena cística en 8 casos $(2 \%)$, basándose en los siguientes parámetros:

1. Sangrado no pulsátil al corte sin drenaje biliar.

2. Ausencia de pulso visible en el extremo grapado.

3. Diámetro similar a la arteria cística.

En 5 casos se decidió tomar una muestra de tejido de la vena cística para estudio histológico el cual resultó con reporte de pared de vena de características usuales. En los otros 3 casos no se tomó muestra para estudio histopatológico debido a que no fue posible obtener tejido con este fin, sin embrago, cumplían los parámetros previamente descritos.

\section{Discusión}

Con el advenimiento de la cirugía laparoscópica y la descripción de la primera serie de casos de colecistectomías laparoscópicas en Colombia en $1991^{16}$, esta se ha convertido en el "gold standard" para la realización de colecistectomías en el mundo y en nuestro país, realizándose aproximadamente el 90\% de los procedimientos con este abordaje ${ }^{17}$. La magnificación de las estructuras ha permitido un mayor detalle de las estructuras anatómicas y evidenciar pequeñas fuentes de sangrado que en cirugía abierta podrían ser insignificantes ${ }^{10}$.

Se han descrito múltiples técnicas para prevenir la lesión de vía biliar las que se encuentran ampliamente descritas y empleadas como lo es la técnica infundibular, disección de vía biliar hasta identificar adecuadamente la inserción del cístico, VCS, colangiografía intraoperatoria, surco de Rouvière, ganglio linfático cístico y técnicas avanzadas de imagen, entre otras ${ }^{15,17}$.

La VCS fue descrita por Strasberg en 1995, en ella se deben cumplir tres requisitos: el primero, retirar la grasa y tejido fibroso del triángulo de Calot, el segundo separar la parte más baja de la vesícula de la placa cística (lecho vesicular) y la tercera es visualizar dos y solo dos estructuras entrando a la vesícula biliar. Cuando estos tres criterios se cumplen se obtiene la $\mathrm{VCS}^{18}$. Sin embargo, en la minoría de los casos no es posible obtenerla como lo hemos observado en 24 años de la descripción de la VCS. Esto no constituye una observación inédita, ya que autores han mencionado la presencia de conductos biliares aberrantes dentro del triángulo hepatocístico ${ }^{19}$ y de arterias císticas múltiples ${ }^{20}$, pero no se ha insistido en la posibilidad de que la vena cística aparezca como un elemento significativo alterando la VCS (Figura 2). Por esta razón, es muy importante tenerla en cuenta en el triángulo hepatocístico para usar otras estrategias y así evitar lesiones de la vía biliar.

Por otro lado, ha sido descrita como una estructura de referencia para prevención de lesiones de vía biliar debido a que se encuentra entre el infundíbulo de la vesícula biliar y la unión del cístico con la vía biliar sin cruzar la vía biliar, por lo cual una disección por encima de esta estructura sería segura ${ }^{10,15}$, aunque de acuerdo a nuestra experiencia no tenemos suficientes elementos para afirmar o negar esta condición de marcador anatómico (Figura 3).

También es importante tener en cuenta el diagnóstico de cirrosis debido a que la hipertensión portal puede hacer evidente la estructura de la vena cística con mayor frecuencia ${ }^{13}$ en nuestra serie se muestra que 1 de los casos en la que fue evidente la vena cística tenía esta patología.

Como vimos en este estudio, la vena cística es una estructura anatómica cuya existencia se debe tener en mente por parte del cirujano que realiza la colecistectomía laparoscópica, porque, aunque en nuestra serie como elemento significativo es del $2 \%$, otras series la describen con una frecuencia mucho mayor $^{14,15}$, este fenómeno posiblemente debido a que en este estudio no se tuvieron en cuenta diámetros pequeños que podrían confundirse con adherencias.

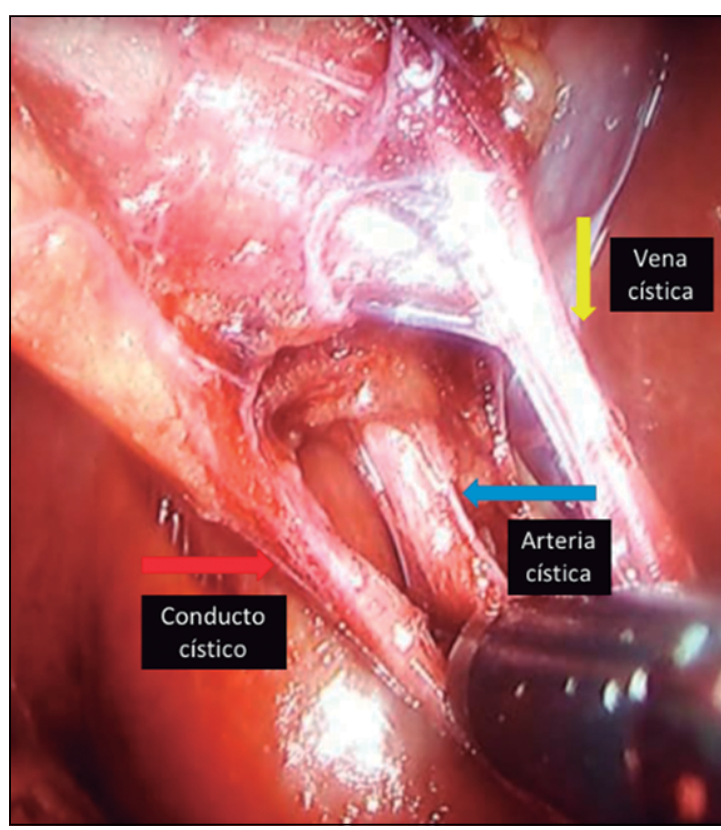

Figura 2. Vena cística de mayor tamaño siendo factor de confusión para obtener una adecuada VCS. 
Figura 3. Pequeña vena cística atravesando triángulo hepatocístico, encontrándose entre el infundíbulo y la unión del conducto cístico con la vía biliar $^{15}$.

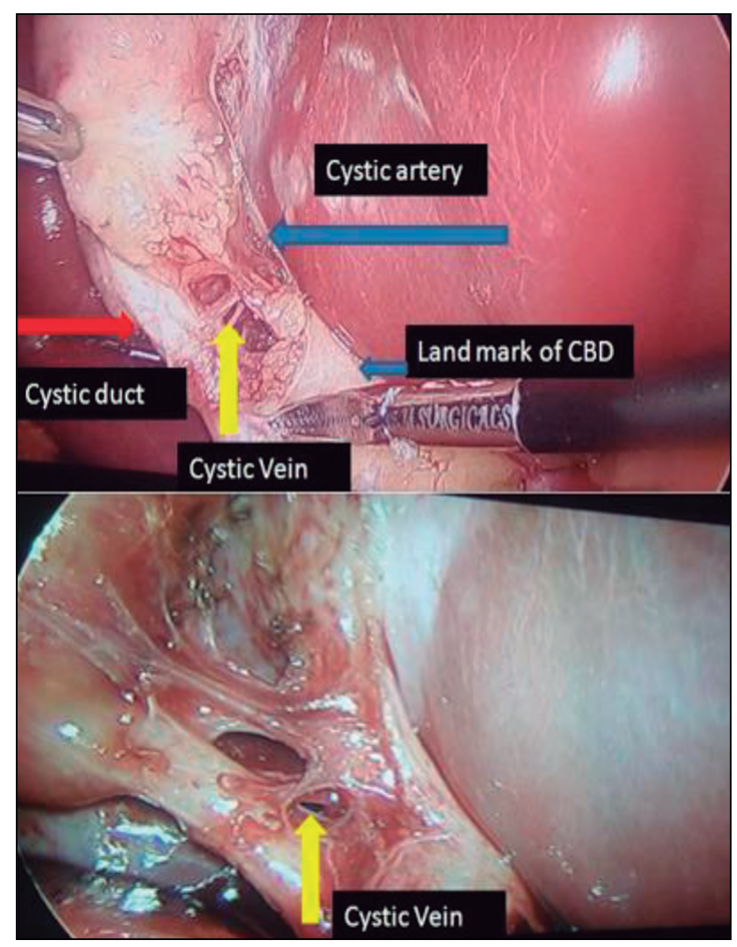

Es necesario realizar más estudios sobre la presencia e importancia como reparo anatómico debido a su valor para prevenir lesiones de la vía biliar y como factor de confusión en la vista crítica de seguridad.
Una de las limitaciones de este estudio es su carácter retrospectivo y la ausencia de estudio histopatológico en 3 de los 8 casos.

\section{Conclusión}

En los libros de anatomía y de cirugía la vena cística no ha tenido una importancia significativa y ha sido documentada de manera superficial. Sin embargo, hemos visto su importancia en un $2 \%$ de los casos para la colecistectomía laparoscópica es necesario desarrollar estudios adicionales en cuanto a su prevalencia y de sus diferentes variantes anatómicas descritas.

\section{$\underline{\text { Responsabilidades éticas }}$}

Protección de personas y animales. Los autores declaran que para esta investigación no se han realizado experimentos en seres humanos ni en animales.

Confidencialidad de los datos. Los autores declaran que en este artículo no aparecen datos de pacientes.

Conflictos de interés: no hay.

\section{Bibliografía}

1. Standring S. Gallbladder and biliary tree. In: Standring S, editor. Gray's Anatomy. 41th ed. Reino Unido: Elsevier; 2015. p. $1173-8$.

2. Skandalakis J, Branum G, Colborn G, Weidman T, Skandalakis P, Skandalakis L. Vías biliares extrahepáticas y vesícula biliar. In: Skandalakis JE, editor. Skandalakis' Bases de la Anatomía Quirúrgica. 1st ed. Madrid: Marbán; 2015. p. 872-6.

3. Keplinger KM, Bloomston M. Anatomy and Embryology of the Biliary Tract. Surg Clin NA [Internet]. 2014;94:20317. Available from: http://dx.doi. org/10.1016/j.suc.2014.01.001.

4. Paquet K. Appraisal of surgical resection of gallbladder carcinoma with special reference to hepatic resection. J HBP Surg. 1998;5:200-6.

5. Abdel-misih SRZ, Bloomston M. Liver
Anatomy Liver Anatomy Surgery Hepatic vasculature Biliary tree. Surg Clin NA [Internet]. 2010;90:643-53. Available from: http://dx.doi.org/10.1016/j. suc.2010.04.017.

6. Bouchet A, Cuilleret J. Hígado y vías biliares. In: Anatomía descriptiva, topográfica y funcional Abdomen. 1st ed. Buenos Aires: Panamericana; 1987. p. 214-56.

7. Durack J, Kohi M. Venous Anatomy of the Abdomen and Pelvis. In: Ho V, Reddy G, editors. Cardiovascular Imaging. 1st ed. St. Louis, Missouri: Elsevier; 2010. p. 1005-18.

8. Boileau Grant J. Abdomen. In: Agur A, Dalley, editors. Grant's Atlas of Anatomy. 12th ed. Baltimore: Williams and Wilkins; 1982. p. 104-95.

9. Moore KL. Abdomen. In: Moore KL, Dalley AF, editors. Anatomía con orientación clínica. 5 th. México: Panamericana; 2007. p. 302-3.
10. Fine A. The Cystic Vein: The Significance of a Forgotten Anatomic Landmark. J Soc Laparoendosc Surg. 1997;1:263-6.

11. Jackson P, Evans S. Biliary system. In: Townsend C, Beauchamp D, editors. Sabiston Textbook of Surgery. 19th ed. Philadelphia: Elsevier; 2012. p. 1476-513.

12. Pham T, Hunter J. Gallbladder and the extrahepatic biliary system. In: Brunicardi C, editor. Schwartz's Principles of Surgery. 10th ed. New York: McGrawHill; 1994. p. 1309-39.

13. Cerril GG, Machado MCC, Pinotti HW, Magalh A. Doppler demonstration of cystic vein dilatation secondary to portal vein thrombosis. Surg Endosc. 1991;5:923 .

14. Satoh T. Study of the anatomy of the venous drainage of the gallbladder. Nagasaki Univ Sch Med. 1989;3:227-33.

15. Samal D, Sahoo R, Mishra SP, Maiti KB, Patra K, Sahu MC. Cystic vein: a guide 
for safer laparoscopic cholecystectomy. Int Surg J. 2017;4:3238-41.

16. Cuéllar C, Moreno E, Casas C, De Francisco J, Ordóñez H, Riveros R. Colecistectomía laparoscópica. Primera experiencia en Colombia. Rev Colomb Cir. 1991;6:5-12.

17. Rose JB, Hawkins WG. Diagnosis and management of biliary injuries. Curr Probl
Surg [Internet]. 2017;54:406-35. Available from: http://dx.doi.org/10.1067/j. cpsurg.2017.06.001.

18. Strasberg SM, Brunt LM. Rationale and Use of the Critical View of Safety in Laparoscopic Cholecystectomy. Am Coll Surg [Internet]. 2010;211:132-8. Available from: http://dx.doi.org/10.1016/j. jamcollsurg.2010.02.053.
19. Skandalakis JE, Gray SW, Rowe JS. Complicaciones Anatómicas en Cirugía General. México: McGraw-Hill; 1996. $134 \mathrm{p}$.

20. Algieri RD, Ferrante MS, Ugartemendía JS, Bernadou M de las M, Pina L, Álvarez A. Trígono Cistohepático: Área Crítica Anatómica para la Seguridad Quirúrgica. Int J Morphol. 2014;32:860-5. 